\title{
Impact of information technology in increasing quality of data in disease registries
}

\author{
Type of article: conference abstract \\ Farzad Mirzakhani*1, Zahra Rahmatinejad1 \\ 1. MSc Student of Medical Informatics, Medical Informatics Department, Faculty of \\ Medicine, Mashhad University of Medical Sciences, Mashhad, Iran \\ *Mirzakhanif921@mums.ac.ir
}

\begin{abstract}
Introduction: The Disease Registry records and maintains the necessary data for each individual patient according to the purpose for which it was designed. These data are widely used to calculate statistical indicators, resource management, resource allocation, and clinical research. One of the main challenges in using disease registries is their low quality data. Incompleteness, inaccuracy and untimeliness are some of the problems with the quality of the data in the disease registries. In this study, the researchers reviewed the views of some medical specialists on ways to improve the quality of data in the diseases registries.In this study, researchers looked at the views of some medical specialist who were in charge of maintaining a disease registry on ways to improve the quality of data in disease registries.

Methods: The qualitative method of in-depth interviews was used to explore the views of medical specialist. They were specialist doctors who were responsible for keeping a disease registry in their specialty. All interviews were conducted by one of the researchers. All interviews were conducted by one of the researchers and the interviews were digitally recorded during the interview. After the interview, the sounds recorded by the researcher were written in the form of text and the themes discussed in them were coded and analyzed. Eventually the coding was reviewed by another researcher, and the opposing opinions of the researchers were resolved through the discussion.

Results: The number of medical specialists interviewed was 6, which all of them was internist. The minimum and maximum interview time was 15 and 35 minutes respectively. The most important solutions to improve the quality of data in the diseases registries are: utilization of software systems (suggested by all doctors), utilization of coded data like icd-10 code (suggested by 5 doctors) and connecting disease registries to hospital information systems and other health information system (suggested by 3 doctors).

Conclusion: According to the views of the medical specialists who participated in present study, the use of IT-based methods as well as information management methods is the best way to improve the quality of data in the registry.
\end{abstract}

Keywords: Disease registry, Data quality, Qualitative study

\section{Declaration of conflicts}

This abstract is selected from the First International Congress of Diseases and Health Outcomes Registry and First National Congress of Medical Informatics, 14-17 February 2017, Mashhad, Iran

\section{Authors' biography}

No biography.

\section{References}

No references. 